\title{
THE ARGHIMEDEAN PROPERTY IN AN ORDERED SEMIGROUP
}

\author{
TÔRU SAITÔ \\ (Received 20 March 1967)
}

\section{Introduction}

By an ordered semigroup we mean a semigroup with a simple order which is compatible with the semigroup operation. Several authors, for example Alimov [1], Clifford [2], Conrad [4] and Hion [7], studied the archimedean property in some special kinds of ordered semigroups. For a general ordered semigroup, Fuchs [6] defined the archimedean equivalence as follows:

$$
\begin{aligned}
& a \sim b \text { if and only if one of the four conditions } \\
& \qquad a \leqq b \leqq a^{n}, \quad b \leqq a \leqq b^{n}, \quad a^{n} \leqq b \leqq a, \quad b^{n} \leqq a \leqq b
\end{aligned}
$$

holds for some positive integer $n$.

Then he mentioned that this relation is an equivalence relation. But this is not correct. In fact, let $S=\{0, a, b\}$ with the product $x y=0$ for every $x, y \in S$ and with the order $a<0<b$. Then it is easily checked that $S$ is an ordered semigroup and that $a \sim 0$ and $b \sim 0$. However, $a \sim b$ does not hold. It seems to be troublesome to define the archimedean equivalence suitably in a general ordered semigroup. In the present note, we restrict our attention to nonnegatively ordered semigroups in the sense defined in $\S 1$. We define the archimedean equivalence in natural way. Even in these semigroups, the archimedean equivalence is not always a congruence relation. The main purpose of $\S 2$ is to give necessary and sufficient conditions in order that the archimedean equivalence is a congruence relation. Such a nonnegatively ordered semigroup is called $a$-regular. Many ordered semigroups, for example all nonnegatively ordered commutative semigroups and the nonnegative cones of all ordered inverse semigroups are $a$-regular. In $\S 3$, we study the structure of $a$-regular nonnegatively ordered semigroups $P$. The quotient semigroup of $P$ modulo the archimedean equivalence is an ordered idempotent semigroup, whose structure was completely determined in our previous paper [8]. By the aid of this knowledge, we show, in this note, the structure of $P$ is known to some extent. 


\section{Preliminaries}

By an ordered semigroup, we mean a semigroup $S$ with a simple order which satisfies

$$
a \leqq b \text { implies } a c \leqq b c \text { and } c a \leqq c b \text { for every } c \in S .
$$

An element $c$ of $S$ is said to lie between $a$ and $b$ if either $a \leqq c \leqq b$ or $b \leqq c \leqq a$. A subset $T$ of $S$ is called convex if $T$ contains with two of its elements all elements of $S$ which lie between them. An element $p$ of $S$ is called positive if $p^{2}>p$, while $q$ is called negative if $q^{2}<q$. Since the order is simple, an element $p$ of $S$ is nonnegative if and only if $p^{2} \geqq p$. An element $p$ of $S$ is called positive (nonnegative) in the strict sense if $p s>s$ and $s p>s$ ( $p s \geqq s$ and $s p \geqq s$ ) for every $s \in S$. Clearly if $p$ is positive (nonnegative) in the strict sense, then $p$ is positive (nonnegative). An ordered semigroup $S$ is called positively (nonnegatively) ordered (in the strict sense), if every element of $S$ is positive (nonnegative) (in the strict sense). The number of distinct powers of an element $a$ of an ordered semigroup $S$ is called the order of $a$. A mapping of an ordered semigroup $S$ into an ordered semigroup $T$ is called an o-isomorphism, if it is a semigroup-isomorphism and an orderisomorphism at the same time. If there is an $o$-isomorphism of $S$ onto $T$, then we say that $S$ is o-isomorphic to $T$.

Now we give some lemmas which we need in the following sections.

Lemma 1.1 ([9] Lemma 1 and its Corollary). The set $P$ of nonnegative elements of an ordered semigroup $S$, if it is nonvoid, is a subsemigroup of $S$. The set $E$ of idempotents of $S$, if it is nonvoid, is a subsemigroup of $S$.

The set $P$ of nonnegative elements of $S$ is called the non-negative cone of $S$. If the set $E$ of idempotents of $S$ is nonvoid, we denote by $\mathscr{D}_{E}$ the $\mathscr{D}$ equivalence in the semigroup $E$, in order to distinguish it from that in the original semigroup $S$.

LEMMA 1.2 ([9] Lemma 2). In an ordered semigroup $S$, if $p$ is nonnegative and $q$ is nonpositive and it $p \leqq q$, then both $p q$ and $q p$ are idempotents which lie between $p$ and $q$.

LEMMA 1.3. An idempotent semigroup $S$ is a semilattice of rectangular bands. Every rectangular band which is a constituent of the decomposition is a $\mathscr{D}$-class of $S$.

The first half of the above Lemma was given in [2] Exercise 1 for $\S 4.2$. Then the second half can be shown easily.

Lemma 1.4 ([8] Theorem 1). In an ordered idempotent semigroup $S$, each $\mathscr{D}$-class consists of either only one $\mathscr{L}$-class or only one $\mathscr{R}$-class.

A $\mathscr{D}$-class of an ordered idempotent semigroup $S$ which consists of only one $\mathscr{L}$-class ( $\mathscr{R}$-class) is called a $\mathscr{D}$-class of $\mathscr{L}$-type ( $\mathscr{R}$-type). By Lemma 1.3, 
the set of $\mathscr{D}$-classes of an ordered idempotent semigroup $S$ forms a semilattice, which is called the associated semilattice of $S$. In the associated semilattice, we denote the partial order by $\leqslant$ and the semilattice operation by $\circ$.

LEMMA 1.5 ([8] Theorem 3). The associated semilattice $S^{*}$ of an ordered idempotent semigroup $S$ is a tree semilattice, i.e. a semilattice in which $\{\xi ; \xi \leqslant \alpha\}$ forms a simply ordered set for every $\alpha \in S^{*}$.

In the tree semilattice $S^{*}, \alpha \in S^{*}$ is called a branching element of $S^{*}$, if there exist $\beta$ and $\gamma$ such that $\alpha \prec \beta, \alpha \prec \gamma$ and $\alpha=\beta \circ \gamma$.

Finally we give the following well-known lemma, which is implicitly included in [5] Théorème 3 in p. 179.

LEMMA 1.6. Let $S$ be an ordered semigroup and let $\rho$ be a congruence relation on $S$ such that every $\rho$-class is convex. For $\rho$-classes $A$ and $B$, we define $A \leqq B$ if and only if $a \leqq b$ for some $a \in A$ and $b \in B$. Then the quotient semigroup $S / \rho$ is an ordered semigroup. Moreover, if $A<B$, then $a<b$ for every $a \in A$ and $b \in B$.

\section{The archimedean equivalence}

In what follows, we always denote by $P$ a nonnegatively ordered semigroup and by $E$ the set of idempotents of $P$. For $x, y \in P$, we define the archimedean equivalence $\sim$ as follows:

$x \sim y$ if and only if $x \leqq y \leqq x^{n}$ or $y \leqq x \leqq y^{n}$ for some positive integer $n$.

LEMMA 2.1. The archimedean equivalence in $P$ is an equivalence relation.

Proof. It suffices to prove only the transitivity. Let $a \sim b$ and $b \sim c$. Then

(1) if $a \leqq b \leqq a^{n}$ and $b \leqq c \leqq b^{m}$, then $a \leqq b \leqq c \leqq b^{m} \leqq a^{m n}$;

(2) if $a \leqq b \leqq a^{n}$ and $c \leqq b \leqq c^{m}$, then, according as $a \leqq c$ or $c \leqq a$, we have $a \leqq c \leqq b \leqq a^{n}$ or $c \leqq a \leqq b \leqq c^{m}$;

(3) if $b \leqq a \leqq b^{n}$ and $b \leqq c \leqq b^{m}$, then, according as $a \leqq c$ or $c \leqq a$, we have $a \leqq c \leqq b^{m} \leqq a^{m}$ or $c \leqq a \leqq b^{n} \leqq c^{n}$;

(4) if $b \leqq a \leqq b^{n}$ and $c \leqq b \leqq c^{m}$, then $c \leqq b \leqq a \leqq b^{n} \leqq c^{m n}$.

Thus, in all cases, we have $a \sim c$.

An equivalence class of $P$ modulo the archimedean equivalence $\sim$ is called an archimedean class.

LEMмA 2.2. Each archimedean class of $P$ is a convex subsemigroup of $P$ which is nonnegatively ordered in the strict sense. 
Proof. Let $A$ be an archimedean class of $P$ and let $a, b \in A$ and $a \leqq c \leqq b$. Since $a \sim b$, we have $b \leqq a \leqq b^{n}$ or $a \leqq b \leqq a^{n}$. If $b \leqq a \leqq b^{n}$, then $a=b=c$, and if $a \leqq b \leqq a^{n}$, then $a \leqq c \leqq b \leqq a^{n}$. Thus, in both cases, we have $a \sim c$ and so $A$ is convex. Next we suppose that $a, b \in A$. Then, since $a \sim b$, we have $a \leqq b \leqq a^{n}$ or $b \leqq a \leqq b^{n}$. If $a \leqq b \leqq a^{n}$, then $a \leqq a^{2} \leqq a b \leqq a^{n+1}$ and so $a \sim a b$. If $b \leqq a \leqq b^{n}$, then $b \leqq b^{2} \leqq a b \leqq$ $b^{n+1}$ and so $b \sim a b$. Thus, in both cases, we have $a b \in A$ and so $A$ is a subsemigroup. Finally, by way of contradiction, we suppose that $a b<a$ for some $a, b \in A$. Then we have $a b^{2} \leqq a b$. On the other hand, since $b \leqq b^{2}$, we have $a b \leqq a b^{2}$. Hence $a b=a b^{2}$ and so $a b=a b^{n}$ for every positive integer $n$. Since $a b<a \leqq a^{2}$, we have $b<a$. But $a \sim b$ and so $b<a \leqq b^{m}$ for some positive integer $m$. Hence $a \leqq a^{2} \leqq a b^{m}=a b<a$, which is a contradiction. Thus $a \leqq a b$ for every $a, b \in A$. Similarly we can prove $a \leqq b a$. Thus $A$ is nonnegative in the strict sense.

Lemma 2.3. For an archimedean class $A$ of $P$, the following conditions are equivalent to one another:

(1) A contains an idempotent,

(2) A has the greatest element,

(3) A has the zero element,

(4) every element of $A$ is an element of finite order,

(5) A contains an element of finite order.

Moreover, under these conditions, an idempotent of $A$ is the greatest element and also the zero element of $A$.

Proof. (1) implies (2). In fact, let $e$ be an idempotent of $A$ and let $a \in A$. Then we wave $a \leqq e \leqq a^{n}$ or $e \leqq a \leqq e^{n}=e$. Thus, in both cases, we have $a \leqq e$. Incidentally we have shown that an idempotent of $A$ is the greatest element of $A$. (2) implies (3). In fact, let $g$ be the greatest element of $A$ and let $a \in A$. By Lemma 2.2, we have $g \leqq g a$ and $g \leqq a g$, and also $a g \in A$ and $g a \in A$ and so $g a \leqq g$ and $a g \leqq g$. Thus $g a=a g=g$. Incidentally we have shown that the greatest element of $A$ is the zero element of $A$. (3) implies (4). In fact, let $A$ have the zero element 0 and let $a \in A$. Then $0 \leqq a \leqq 0^{n}=0$ or $a \leqq 0 \leqq a^{n}$. In the former case, we have $a=0$ and $a=a^{2}$. In the latter case, we have $0 \leqq a^{n} \leqq 0^{n}=0$ and so $a^{n}=0$ and $a^{n}=a^{n+1}$. (4) implies (5) trivially. Finally (5) implies (1). In fact, let $a$ be an element of finite order in $A$. Then $a^{n}=a^{n+1}$ for some positive integer $n$, and $a^{n}$ is an idempotent of $A$.

Corollary 2.4. Every archimedean class of $P$ contains at most one idempotent.

If an archimedean class $A$ satisfies any one of the conditions in Lemma 
2.3, then $A$ is called a periodic archimedean class. Otherwise $A$ is called a nomperiodic archimedean class.

Lemma 2.5. In $P$, each nonperiodic archimedean class $A$ is positively ordered in the strict sense.

Proof. By Lemma 2.2, we have $a \leqq a b$ for every $a, b \in A$. Now, by way of contradiction, we assume that $a=a b$. Then we have $a=a b^{m}$ for every positive integer $m$. Since $a \sim b$, we have either $a \leqq b \leqq a^{n}$ or $b \leqq a \leqq b^{n}$. If $b \leqq a \leqq b^{n}$, then $a^{2} \leqq a b^{n}=a \leqq a^{2}$ and so $a=a^{2}$. If $a \leqq b \leqq a^{n}$, then $a^{n} \leqq b^{n}$ and so $a \leqq a^{2} \leqq a^{n+1} \leqq a b^{n}=a$ and $a=a^{2}$. Hence, in both cases, $a$ is an jdempotent of $A$, which contradicts that $A$ is non-periodic. Thus we have $a<a b$. We can prove $a<b a$ in a similar way.

EXAMPLE 2.6. Let $K_{1}=\{e, f, a, g\}$ be a system with the multiplication table

\begin{tabular}{l|llll} 
& $e$ & $f$ & $a$ & $g$ \\
\hline$e$ & $e$ & $e$ & $e$ & $e$ \\
$f$ & $f$ & $f$ & $f$ & $f$ \\
$a$ & $f$ & $g$ & $g$ & $g$ \\
$g$ & $g$ & $g$ & $g$ & $g$
\end{tabular}

and with the order $e<t<a<g$. It is easily checked that $K_{1}$ is an ordered semigroup.

ExAMPLE 2.7. Let $K_{2}=\{e, f, a, g\}$ be an ordered semigroup with the product multiplicatively dual to that of $K_{1}$ and with the same order relation as $K_{1}$.

THEOREM 2.8. In order that the archimedean equivalence in a nonnegatively ordered semigroup $P$ is not a congruence relation, it is necessary and sufficient that $P$ contains a subsemigroup o-isomorphic to either $K_{1}$ or $K_{2}$ in the above Examples.

Proof. Necessity. Let the archimedean equivalence $\sim$ in $P$ be not a congruence relation. Then there exist elements $a, b, c \in P$ such that $a \sim b$ but either $a c \sim b c$ or $c a \sim c b$ does not hold. First we consider the case when $a c \sim b c$ does not hold and suppose without loss of generality that $a \leqq b \leqq a^{n}$. Then $a c \leqq b c \leqq a^{n} c$ and, since $a c \neq b c$, we have $n>1$. Now we give a series of relations which hold for $a, b$ and $c$.

-(1) $(a c)^{m}<$ a for every positive integer $m$.

In fact, if $(a c)^{m} \geqq a$ for some $m$, then

$$
a^{n} c=a^{n-1}(a c) \leqq(a c)^{m(n-1)}(a c)=(a c)^{m(n-1)+1} .
$$


Hence we have $a c \leqq b c \leqq a^{n} c \leqq(a c)^{m(n-1)+1}$ which contradicts that $a c \sim b c$ does not hold.

(2) $a c<a$.

The special case of (1) for $m=1$.

(3) $a c^{m}=a c$ for every positive integer $m$.

In fact, by (2), we have $a c^{2} \leqq a c$. On the other hand, since $c \leqq c^{2}$, we have $a c \leqq a c^{2}$. Hence $a c=a c^{2}$ and so $a c=a c^{m}$.

(4) $c a<a c$.

In fact, if $a c \leqq c a$, then, by (3), we have $a^{n} c=a^{n} c^{n} \leqq(a c)^{n}$. Hence $a c \leqq b c \leqq a^{n} c \leqq(a c)^{n}$, which contradicts that $a c \sim b c$ does not hold.

(5) $c a=c a c$.

In fact, by (4), we have $c a \leqq c^{2} a=c(c a) \leqq c a c$. On the other hand, by (2), we have $c a c \leqq c a$. Hence we have $c a=c a c$.

(6) $a<a^{2} c$.

In fact, if $a^{2} c \leqq a$, then, by (3), we have $a^{2} c=a^{2} c^{2}=\left(a^{2} c\right) c \leqq a c$. On the other hand, since $a \leqq a^{2}$, we have $a c \leqq a^{2} c$. Hence $a c=a^{2} c$ and so $a c=a^{n} c$. Therefore $a c \leqq b c \leqq a^{n} c=a c$, which contradicts that $a c \sim b c$ does not hold.

(7) $a c a<a$.

In fact, by (5) and (1), we have aca $=a c a c=(a c)^{2}<a$.

(8) $(a c)^{2}=a c,(c a)^{2}=c a$.

In fact, by (7), we have $(a c)^{2}=a c a c \leqq a c$ and $(c a)^{2}=c a c a \leqq c a$. On the other hand, since $a c$ and $c a$ are nonnegative, these elements are idempotents.

(9) $\left(a^{2} c\right)^{2}=a^{2} c=a^{2}$.

In fact, by (5) and (8), we have

$$
\left(a^{2} c\right)^{2}=a^{2}(c a) a c=a^{2}(c a c) a c=a(a c)^{3}=a(a c)=a^{2} c .
$$

Hence, by (6) and (2), we have $a^{2} \leqq\left(a^{2} c\right)^{2}=a^{2} c \leqq a^{2}$ and so $\left(a^{2} c\right)^{2}=$ $a^{2} c=a^{2}$.

Now we put $c a=e, a c=f, a^{2}=a^{2} c=g$. Then, by (4), (2) and (6), we have $e<f<a<g$. Moreover

$$
e=e^{2} \leqq e f \leqq e a \leqq e g=(c a) a a=(c a c) a a=c a(c a c) a=(c a)^{3}=c a=e
$$
by (8) and (5),

$$
f=a c=a c a c=a(c a)=a c^{2} a=t e \leqq f^{2} \leqq t a \leqq t g=a c a a=a c a c a=
$$
acacac $=(a c)^{3}=a c=f$ by $(8),(5)$ and $(3)$,

$$
\begin{aligned}
& f=a c=a c a c=a c a=a e \text { by }(8) \text { and }(5), \\
& g=a^{2} c=a f \leqq a^{2} \leqq a g=a a^{2}=a^{3}=g \text { by }(9),
\end{aligned}
$$


$g=a(a c)=a(a c)^{2}=a^{2} c a c=a^{2}(c a)=g e \leqq g f \leqq g a \leqq g^{2}=\left(a^{2} c\right)^{2}=$ $a^{2} c=g$ by (8), (5) and (9).

Thus the set consisting of four elements $e, f, a$ and $g$ forms a subsemigroup $o$-isomorphic to $K_{1}$. In the case when $c a \sim c b$ does not hold we can prove similarly that $P$ contains a subsemigroup $o$-isomorphic to $K_{2}$.

Sufficiency. We suppose that $P$ contains a subsemigroup $o$-isomorphic to $K_{1}$. Without loss of generality, we assume $P$ contains the ordered semigroup $K_{1}$. Then, since $a^{2}=g$, we have $a \sim g$. But $a e=f, g e=g$ and so $a e \sim$ ge does not hold. Thus the archimedean equivalence is not a congruence relation. In the case when $P$ contains a subsemigroup $o$-isomorphic to $K_{2}$, we can obtain the same conclusion in a similar way.

A nonnegatively ordered semigroup $P$ is called a-regular if the archimedean equivalence in $P$ is a congruence relation.

COROLLARY 2.9. A nonnegatively ordered semigroup $P$ is a-regular if one of the following conditions is satisfied:

(1) $P$ is commutative,

(2) $P$ contains no elements of finite order except idempotents,

(3) $P$ is the nonnegative cone of an ordered inverse semigroup.

Proof. In cases (1) and (2), it is trivial that $P$ does not contain a subsemigroup $o$-isomorphic to $K_{1}$ or $K_{2}$. Since an ordered inverse semigroup contains no elements of finite order except idempotents ([9] Theorem 6), the case (3) is reduced to the case (2).

REMARK. When $P$ is the nonnegative cone of an ordered regular semigroup which contains a non-idempotent element of finite order, then, by [9] Theorems 2 and 3, $P$ contains a subsemigroup 0 -isomorphic to $K_{1}$ or $K_{2}$. Hence $P$ is not $a$-regular.

THEOREM 2.10. A nonnegatively ordered semigroup $P$ is a-regular if and only if it satisfies the condition

(a) $\quad a \sim g=g^{2}, e=e^{2}<g$ and $e \mathscr{D}_{E} g$ imply either $e a=g$ or $a e=g$.

Proof. Let $P$ be $a$-regular and let $a \sim g=g^{2}, e=e^{2}<g$ and $e \mathscr{D}_{E} g$. Then, by Lemma 2.3 , we have $a \leqq g$. Now we have also $e<a$. In fact, otherwise, $a \leqq e<g$ and so, by Lemma 2.2, we have $e \sim g$, which contradicts Corollary 2.4. First we suppose that the $\mathscr{D}_{E^{-}}$-lass of $E$ which contains $e$ is of $\mathscr{L}$-type. Then $e=e^{2} \leqq e a \leqq e g=e$. Hence we have $e a=e$. Therefore $(a e)^{2}=a e a e=a e$ and so $a e$ is an idempotent. Since $\sim$ is a congruence relation, we have $a e \sim g e=g$. Hence, by Corollary 2.4 , we have $a e=g$. If the $\mathscr{D}_{E}$-class which contains $e$ is of $\mathscr{R}$-type, we can prove $e a=g$ in a similar way. Conversely we suppose that $P$ is not $a$-regular. Then, by Theorem 2.8, 
$P$ contains a subsemigroup $o$-isomorphic to either $K_{1}$ or $K_{2}$. If $P$ contains $K_{1}$, then three elements $e, a$ and $g$ of $K_{1}$ satisfy the assumption of the condition $(\alpha)$. But we have $e a=e \neq g$ and $a e=f \neq g$ and so the condition $(\alpha)$ does not hold. If $P$ contains $K_{2}$, we can obtain the same conclusion in a similar way.

\section{3. a-regular nonnegatively ordered semigroups}

In this section, we denote by $P$ an $a$-regular nonnegatively ordered semigroup and by $A(p)$ the archimedean class which contains an element $p \in P$. Since $P$ is $a$-regular, the archimedean equivalence $\sim$ is a congruence relation and so, by Lemmas 2.2 and 1.6, the quotient semigroup $P / \sim$ is an ordered semigroup with the order defined in Lemma 1.6. We denote by $\bar{P}$ the ordered semigroup $P / \sim$.

Theorem 3.1. $\bar{P}$ is an ordered idempotent semigroup.

Proof. Let $A(p)$ be an element of $\bar{P}$. Then, since $p \sim p^{2}$, we have $(A(p))^{2}=A\left(p^{2}\right)=A(p)$.

Lemma 3.2. The mapping $\varphi$ which maps $e \in E$ to $A(e) \in \bar{P}$ is an o-isomorphism of $E$ into $\bar{P}$.

Proof. By Corollary 2.4, $\varphi$ is a one-to-one mapping. Then it is easily seen that $\varphi$ is a semigroup-isomorphism and an order-isomorphism.

The image set of the $o$-isomorphism $\varphi$ in the above Lemma 3.2 is denoted by $\bar{E} . \bar{E}$ is a subsemigroup of $\bar{P}$. For an archimedean class $A$, we have $A \in \bar{E}$ if and only if $A$ contains an idempotent. Hence $\bar{E}$ is the set of periodic archimedean classes. The $\mathscr{D}$-equivalence in the ordered idempotent semigroup $\bar{P}$ is denoted by $\overline{\mathscr{D}}$. For $A \in \bar{P}$, the $\overline{\mathscr{D}}$-class which contains $A$ is denoted by $\overline{\mathscr{D}}(A)$.

Theorem 3.3. If $A \in \bar{E}$, then $\overline{\mathscr{D}}(A) \subseteq \bar{E}$.

Proof. Let $B \in \bar{P}$ such that $A \overline{\mathscr{D}} B$. First we suppose that $\overline{\mathscr{D}}(A)$ is a $\overline{\mathscr{D}}$-class of $\mathscr{L}$-type. Since $A \in \bar{E}, A$ contains an element $e \in E$. We take $b \in B$ arbitrarily. If $b \leqq e$, then, by Lemma $1.2, b e$ is an idempotent of $P$ and $b e \in$ $B A=B$. If $e \leqq b$, then we have $e=e^{2} \leqq e b \in A B=A$. Hence, by Lemma 2.3 , we have $e=e b$ and so $(b e)^{2}=b e b e=b e$ and $b e \in B A=B$. Hence $b e$ is an idempotent of $B$. Thus, in both cases, we obtain $B \in \bar{E}$. In the case when $\overline{\mathscr{D}}(A)$ is of $\mathscr{R}$-type, we can prove $B \in \bar{E}$ in a similar way.

By Theorem 3.3, each $\overline{\mathscr{D}}$-class $\bar{D}$ in $\bar{P}$ belongs to one and only one of the following two types:

(1) all archimedean classes in $\bar{D}$ are periodic,

(2) all archimedean classes in $\bar{D}$ are nonperiodic. 
If a $\overline{\mathscr{D}}$-class $\bar{D}$ belongs to the type (1), then $\bar{D}$ is called a periodic $\overline{\mathscr{D}}$-class, while if $\bar{D}$ belongs to the type (2), it is called a nonperiodic $\overline{\mathscr{D}}$-class.

THEOREM 3.4. If $A$ is an archimedean class which belongs to a periodic $\bar{D}$-class $\bar{D}$ and if $A$ is not the least element of $\bar{D}$ with respect to the order in $\bar{P}$, then, in $P$, every element of $A$ is at most of order 2.

Proof. Let $a \in A$. By assumption, there exists an archimedean class $B \in \bar{D}$ such that $B<A$. Since $\bar{D}$ is a periodic $\overline{\mathscr{D}}$-class, both $A$ and $B$ are periodic archimedean classes. Let $e$ and $f$ be idempotents of $A$ and $B$, respectively. Then, since $B<A$, we have $f<a \leqq e$. First we suppose that the $\overline{\mathscr{D}}$-class $\bar{D}$ is of $\mathscr{L}$-type. Then ef $\in A B=A$ and $f e \in B A=B$. Since $e f \in E$ and $f e \in E$, we have $e f=e$ and $f e=f$ by Corollary 2.4, and so $e \mathscr{D}_{E} f$. In the case when $\bar{D}$ is of $\mathscr{R}$-type, we can prove $e \mathscr{D}_{E} f$ in a similar way. Hence, in both cases, by Theorem 2.10 , we have $f a=e$ or $a f=e$. On the other hand, since $f<a \leqq e$, we have $f a \leqq a^{2} \leqq e^{2}=e$ and $a f \leqq a^{2} \leqq e^{2}=e$. Therefore we have $a^{2}=e$.

Theorem 3.5. Suppose that, for $A \in \bar{P}$, there exists $B \in \bar{P}$ such that $A<B$ and $\overline{\mathscr{D}}(A) \leqslant \overline{\mathscr{D}}(B)$. Then $A$ is a periodic archimedean class.

Proof. First we suppose that $\overline{\mathscr{D}}(A)$ is a $\overline{\mathscr{D}}$-class of $\mathscr{L}$-type. Then, since $\overline{\mathscr{D}}(A)=\overline{\mathscr{D}}(A) \circ \overline{\mathscr{D}}(B)=\overline{\mathscr{D}}(A B)$, we have $A B=A(A B)=A$. We take $a \in A$ and $b \in B$ arbitrarily. Then $a b \in A B=A$ and so $a b<b$. Hence we have $a^{2} b \leqq a b$. On the other hand, since $a \leqq a^{2}$, we have $a b \leqq a^{2} b$. Therefore $a b=a^{2} b=a(a b)$ with $a \in A$ and $a b \in A$. Hence, by Lemma 2.5, $A$ is a periodic archimedean class. In the case when $\overline{\mathscr{D}}(A)$ is of $\mathscr{R}$-type, we can obtain the same conclusion in a similar way.

TheOREM 3.6. Every nonperiodic $\overline{\mathscr{D}}$-class $\bar{D}$ consists of only one nonperiodic archimedean class.

Proof. By way of contradiction, we assume that $\bar{D}$ contains two distinct archimedean classes $A$ and $B$. Without loss of generality, we suppose that $A<B$. Then $\overline{\mathscr{D}}(A)=\bar{D}=\overline{\mathscr{D}}(B)$ and $A$ is a nonperiodic archimedean class, which contradicts Theorem 3.5 .

COROLlary 2.7. Let $A$ be a nonperiodic archimedean class and let $B$ be an archimedean class such that $A<B$. Then there exists an archimedean class $C$ such that $A<C$ and $\overline{\mathscr{D}}(A)>\overline{\mathscr{D}}(C)$.

Proof. We put $C=A B$. Then, by Lemma 1.2 , we have $A \leqq C \leqq B$. If it were true that $A=C$, then $A=C<B$ and $\overline{\mathscr{D}}(A)=\overline{\mathscr{D}}(C)=\overline{\mathscr{D}}(A B)$ $\preccurlyeq \overline{\mathscr{D}}(B)$, which contradicts Theorem 3.5. Hence we have $A<C$. Moreover $\overline{\mathscr{D}}(C)=\overline{\mathscr{D}}(A B) \leqslant \overline{\mathscr{D}}(A)$ and the equality is excluded by Theorem 3.6. Thus we have $\overline{\mathscr{D}}(A) \succ \overline{\mathscr{D}}(C)$. 
REMARK. Intuitively speaking, when we pursue the course on the associated semilattice of $\bar{P}$ according to the order, every nonperiodic archimedean class appears in the descending path. In particular, every branching element of the associated semilattice is a periodic $\overline{\mathscr{D}}$-class.

Theorem 3.8. Let $A$ and $B$ be archimedean classes such that $A<B$.

(1) If $A B<B$, then $A B$ is a periodic archimedean class and, for every $a \in A$ and $b \in B$, the product ab is equal to the idempotent of $A B$.

(2) If $B A<B$, then $B A$ is a periodic archimedean class and, for every $a \in A$ and $b \in B$, the product ba is equal to the idempotent of $B A$.

Proof. First we consider (1) and suppose that $A B<B$. Then $\overline{\mathscr{D}}(A B)=$ $\overline{\mathscr{D}}(A) \circ \overline{\mathscr{D}}(B) \leqslant \overline{\mathscr{D}}(B)$ and so, by Theorem $3.5, A B$ is a periodic archimedean class. Let $g$ be the idempotent of $A B$ and let $a \in A$ and $b \in B$. Then, since $A B<B$, we have $g<b$ and so $a g \leqq a b$. On the other hand, by Lemma 2.3, $g$ is the greatest element of $A B$ and $A \leqq A B$. Hence we have $a \leqq g$. Therefore, by Lemma 1.2, ag is an idempotent and also ag $\in A(A B)=A B$. Hence we have $g=a g$. Since $a b \in A B$, we have $a b \leqq g=a g$ by Lemma 2.3 again. Thus $a b=a g=g$. The assertion (2) can be proved in a similar way.

REMARK. If $A B=B$, the product $a b$ varies in general according to the choice of elements $a \in A$ and $b \in B$. For the study of the structure in this case, it needs to discuss beforehand the inner structure of archimedean classes.

\section{Appendix}

I express my hearty thanks to the referee for his suggestions given to this paper.

\section{References}

[1] N. G. Alimov, 'On ordered semigroups', Izv. Akad. Nauk SSSR $14(1950), 569-576$ (Russian).

[2] A. H. Clifford, 'Naturally totally ordered commutative semigroups', Amer. J. Math. 76 (1954), $631-646$.

[3] A. H. Clifford and G. B. Preston, The algebraic theory of semigroups I (Amer. Math. Soc. Math. Surveys No. 7, 1961).

[4] P. Conrad, 'Ordered semigroups', Nagoya Math. J. 16 (1960), 51-64.

[5] M. L. Dubreil-Jacotin, L. Lesieur and R. Croisot, Leçons sur la théorie des treillis, des structures algébriques ordonnées et des treillis géométriques (Gauthier-Villars, 1953).

[6] L. Fuchs, Partially ordered algebraic systems (Pergamon Press, 1963).

[7] Ya. V. Hion, 'Ordered semigroups', Izv. Akad. Nauk SSSR 21 (1957), 209-222 (Russian).

[8] T. Saitô, 'Ordered idempotent semigroups', J. Math. Soc. Japan 14 (1962), 150-169.

[9] T. Saitô, 'Regular elements in an ordered semigroup', Pacific J. Math. 13 (1963), 263-295. Correction, 14 (1964), 1505.

[10] E. Ya. Gabovits, Ordered semigroups (Autoreview of dissertation, Leningrad, 1967) (Russian). 